\title{
A CONFORMAL FIELD THEORY OF
}

\section{EXTRINSIC GEOMETRY OF 2-d SURFACES}

\author{
K.S.Viswanathan円 \\ Department of Physics \\ Simon Fraser University \\ Burnaby V5A 1S6, B.C., Canada. \\ and \\ R.Parthasarathy $\mathrm{I}^{2}$ \\ Institute of Mathematical Sciences \\ Madras 600 113, India.
}

Total number of pages: 30

\footnotetext{
${ }^{1}$ e-mail address: kviswana@sfu.ca

${ }^{2}$ e-mail address: sarathy@imsc.ernet.in
} 
Proposed Running Head

\section{CONFORMAL FIELD THEORY OF EXTRINSIC GEOMETRY}

Name and Mailing Address

K.S.Viswanathan

Department of Physics

Simon Fraser University

Burnaby B.C

Canada V5A 1S6

Telephone: (604) 291 - 3157

Fax: (604) 291 - 3592 
Abstract

In the description of the extrinsic geometry of the string world sheet regarded as a conformal immersion of a 2 -d surface in $R^{3}$, it was previously shown that, restricting to surfaces with $h \sqrt{ } g=1$, where $h$ is the mean scalar curvature and $g$ is the determinant of the induced metric on the surface, leads to Virasaro symmetry. An explicit form of the effective action on such surfaces is constructed in this article which is the extrinsic curvature analog of the WZNW action. This action turns out to be the gauge invariant combination of the actions encountered in 2-d intrinsic gravity theory in light-cone gauge and the geometric action appearing in the quantization of the Virasaro group. This action, besides exhibiting Virasaro symmetry in z-sector, has $S L(2, C)$ conserved currents in the $\bar{z}$-sector. This allows us to quantize this theory in the $\bar{z}$-sector along the lines of the WZNW model. The quantum theory on $h \sqrt{ } g=1$ surfaces in $R^{3}$ is shown to be in the same universality class as the intrinsic 2 -d gravity theory. 


\section{INTRODUCTION}

Extrinsic geometry of surfaces immersed in $R^{n}$ plays an important role in QCD-strings, 3-dimensional Ising model and in the study of biological membranes. We have in our earlier publications $[2,3]$ approached this problem in terms of the Grassmannian $\sigma$-model. In this approach, we make use of the Gauss map [4] of conformally immersed surfaces in $R^{n}$ into the Grassmannian manifold $G_{2, n}$ realized as a quadric in $C P^{n-1}$. This realization plays a key role in the Gauss map. However, in order for the Grassmannian manifold to form tangent planes to a given surface in $R^{n},(n-2)$ integrability conditions must be satisfied by the $G_{2, n}$ fields. These conditions have been explicitely derived in [4] for $R^{3}$ and $R^{4}$ and by us [3] for $R^{n}(n>4)$. The advantage of this approach to the string theory is that one is able to rewrite the string action consisting of both the NambuGoto and extrinsic curvature terms in terms of constrained Kähler $\sigma$ model action. In a subsequent work [5] we have shown that in the geometry of surfaces immersed in $R^{3}$ with the property $h \sqrt{g}=1$, where $h$ is the scalar mean curvature of and $g$ the determinant of the induced metric on the surface, there is a hidden Virasaro symmetry. Specifically, it was shown that $H_{z z} / \sqrt{g}$, where $H_{\alpha \beta}$ are the components of the second fundamental form of the surface, transforms like energy momentum tensor $\left(T_{z z}\right)$ and that $H_{\bar{z} \bar{z}}$ transforms like the metric tensor under the Virasaro transformation. It was further shown that in $R^{4}$ one has analogous properties for surfaces with $h \sqrt{g}=1$, where $\left.h=\sqrt{(} h_{1}^{2}+h_{2}^{2}\right)$, and $h_{1}$ and $h_{2}$ are the two scalar mean curvatures along the two normals to the surface. The hidden symmetry in $R^{4}$ is found to be Virasaro $\otimes$ Virasaro. A simple way to see why one has doubling of the Virasaro algebra in $R^{4}$ is to observe that $G_{2,4} \simeq C P^{1} \otimes C P^{1}$, while $G_{2,3} \simeq C P^{1}$. For surfaces immersed in $R^{n}(n>4)$ not much is known although it is expected that one would find W-gravities in certain type of surfaces [6]. 
In this article, we continue our investigation of $h \sqrt{g}=1$ surfaces in $R^{3}$. We construct an action on such surfaces involving their extrinsic geometric properties. The procedure used here is a variant of the one in [7,8], done in the context of 2 -d intrinsic gravity. In [8], Majorana fermions interacting with zweibein are integrated out to obtain the gravitational WZNW action. Its properties in the light-cone gauge (l.c) are unravelled in [7]. Here, we consider 2-d fermions coupled however, to extrinsic curvature of the surface on which the fermions live, by minimal coupling. The minimal coupling for immersion in $R^{3}$, is through $S O(3)$ gauge fields determined by a local orthonormal frame on the surface. Integrating out the fermions [9], we obtain the WZNW action which in this case may be termed as the induced extrinsic gravity action. This action has local $S O(3)$ gauge invariance. The restriction to $h \sqrt{g}=1$ partially fixes the gauge for $A_{z}^{a},(a= \pm, 0)$ as the gauge fields encode here the information about the extrinsic properties of the immersed surface. Let us note that this restriction is not generally covariant and clearly it is a gauge choice. It is equivalent to an algebraic gauge condition. Our gauge choice is made on surfaces realized in a conformal immersion. In [10], the property of diffeomorphisms arising from partial gauge fixing of $S L(2, R)$ gauge fields was considered. Here, we have found a physical system where these gauge fields and the partial gauge fixing which were ad hoc in [10], arise naturally when treating $h \sqrt{g}=1$ surfaces. The resulting action is given explicitly by

$$
\Gamma_{e f f}\left(F_{1}, F_{2}\right)=\Gamma_{+}\left(F_{2}\right)+\Gamma_{-}\left(F_{1}\right)-\frac{k}{4 \pi} \int\left(\frac{\partial_{\bar{z}} F_{2}}{\partial_{z} F_{2}}\right) D_{z} F_{1} d z \wedge d \bar{z}
$$

where,

$$
D_{z} F_{1}=\frac{\partial_{z}^{3} F_{1}}{\partial_{z} F_{1}}-\frac{3}{2}\left(\frac{\partial_{z}^{2} F_{1}}{\partial_{z} F_{1}}\right)^{2}
$$

is the Schwarz derivative of $F_{1}$. 
In (1), $\Gamma_{+}\left(F_{2}\right)$ is precisely the action that appears in intrinsic 2-d gravity theory in l.c gauge [7], while $\Gamma_{-}\left(F_{1}\right)$ is the geometric action that one encounters in the quantization of the Virasaro group by the method of coadjoint orbits [11]. The coupling term in (1) is needed to make $\Gamma_{\text {eff }}\left(F_{1}, F_{2}\right)$ invariant under Virasaro transformation of $F_{1}$ and $F_{2}$, which are related to the second fundamental form as follows (see section II.);

$$
\frac{\partial_{\bar{z}} F_{2}}{\partial_{z} F_{2}}=H_{\bar{z} \bar{z}}
$$

and

$$
D_{z} F_{1}=H_{z z} / \sqrt{g}
$$

We find that (1) is invariant under Virasaro transformation, whose infinitesimal version reads as

$$
\begin{aligned}
\delta_{\epsilon(\bar{z}, z)}\left(H_{z z} / \sqrt{g}\right) & =-\frac{k}{2} \partial_{z}^{3} \epsilon(\bar{z}, z)-2\left(\partial_{z} \epsilon(\bar{z}, z)\right) H_{z z} / \sqrt{g} \\
& -\epsilon(\bar{z}, z) \partial_{z}\left(H_{z z} / \sqrt{g}\right)
\end{aligned}
$$

and

$$
\delta_{\epsilon(\bar{z}, z)}\left(H_{\bar{z} \bar{z}}\right)=\frac{k}{2} \partial_{\bar{z}} \epsilon(\bar{z}, z)+\epsilon(\bar{z}, z) \partial_{z} H_{\bar{z} \bar{z}}-\left(\partial_{z} \epsilon\right) H_{\bar{z} \bar{z}}
$$

Transformations (5) and (6) are equivalent to the following changes in $F_{i}$,

$$
\delta_{\epsilon} F_{i}=\epsilon(\bar{z}, z) \partial_{z} F_{i} ; \quad(i=1,2)
$$

In what follows we shall call $\Gamma_{+}\left(F_{2}\right)$ as the l.c action, while $\Gamma_{-}\left(F_{1}\right)$ as the geometric action. It should be emphasized here (to avoid any confusion) that we are discussing a 2-d extrinsic gravity theory (extrinsic curvature induced 2-d gravity theory) and $F_{1}$ and $F_{2}$ are related to extrinsic geometry by (3) and (4), while the corresponding action in 2-d 
intrinsic gravity theory is in terms of an $F$ which parameterizes the metric tensor of the 2-d surface.

The equation of motion following from (1) is given by

$$
\partial_{z}^{3} H_{\bar{z} \bar{z}}=\left[\partial_{\bar{z}}-H_{\bar{z} \bar{z}} \partial_{z}-2\left(\partial_{z} H_{\bar{z} \bar{z}}\right)\right] \frac{H_{z z}}{\sqrt{g}} .
$$

This equation is identically satisfied if we take both the first fundamental form (induced metric $\left.g_{\alpha \beta}\right)$ and the second fundamental form $\left(H_{\alpha \beta}\right)$ as determined by $X^{\mu}(\bar{z}, z)$. However, we will see that it is convenient to regard $H_{z z} / \sqrt{g}$ and $H_{\bar{z} \bar{z}}$ as independent fields, independent of $X^{\mu}$, but are related by the equation of motion (8). It is further shown that there is a useful composition formula for (1), which enables us to write it either as l.c action for $F_{2} \circ F_{1}^{-1}=F_{2}\left(\bar{z}, F_{1}^{-1}(\bar{z}, z)\right)$, where the inverse function is defined through

$$
F_{i}\left(\bar{z}, F_{i}^{-1}(\bar{z}, z)\right) \equiv z
$$

or as the geometric action for $F_{1} \circ F_{2}^{-1}$. This important correspondence enables us to find the conserved $S L(2, C)$ currents for the action (1) in the $\bar{z}$ (left) sector of the theory. The total energy momentum tensor $T_{\bar{z} \bar{z}}^{t o t}$ is of the Sugawara form. The theory of extrinsic geometry induced 2-d gravity action for $h \sqrt{g}=1$ surfaces in $R^{3}$ is then quantized in the left sector. From these observations, it is clear that the quantum theory of the left sector on $h \sqrt{g}=1$ surfaces is in the same universality class as the 2-d intrinsic gravity theory.

It is well known that the conventional extrinsic curvature action [1] is given by

$$
S_{P}=\frac{1}{\alpha_{0}^{2}} \int|H|^{2} \sqrt{g} d z \wedge d \bar{z}
$$

It is interesting to note that the third term (interaction term) in (1) is precisely (10). Thus we could say that $(1)$ is the conformally invariant extension of (10) where $H_{z z} / \sqrt{g}$ and $H_{\bar{z} \bar{z}}$ are the dynamical fields. 
It will be shown that $F_{1}$ and $F_{2}$ appearing in (1) are the inhomogeneous coordinates of $G_{2,3} \simeq C P^{1}$ i.e., the Grassmannian $\sigma$-model fields arising in the Gauss map. The paper is organized as follows. In section II we review the basic notions of the Gauss map of surfaces and the properties of $h \sqrt{g}=1$ surfaces. In section III, we derive the Virasaro invariant action for extrinsic geometry induced 2-d gravity. The composition formulae and symmetries of this action in both the left and right sectors are studied in section IV. The theory is quantized in section $\mathrm{V}$ and the results are summarized in section VI.

\section{BASIC PROPERTIES OF $h \sqrt{g}$ SURFACES IN $R^{3}$}

We shall briefly review the basic properties of extrinsic geometry of surfaces in $R^{3}$ [see Ref.2,4 for details] in the following subsections.

(i) Let $X^{\mu}\left(\xi^{1}, \xi^{2}\right),(\mu=1,2,3)$ be the immersion coordinates of a surface $S$ in $R^{3}$. The induced metric on $S$ is

$$
g_{\alpha \beta}=\partial_{\alpha} X^{\mu} \partial_{\beta} X_{\mu} \quad(\alpha, \beta=1,2) .
$$

The second fundamental form $H_{\alpha \beta}$ is defined by

$$
\partial_{\alpha} \partial_{\beta} X^{\mu}=\Gamma_{\alpha \beta}^{\gamma} \partial_{\gamma} X^{\mu}+H_{\alpha \beta} N^{\mu},
$$

and

$$
\partial_{\alpha} N^{\mu}=-H_{\alpha \beta} g^{\beta \gamma} \partial_{\gamma} X^{\mu} .
$$

$\Gamma_{\alpha \beta}^{\gamma}$ are the affine connections determined by the induced metric (11) and $N^{\mu}$ is the local normal to $S$. The intrinsic scalar curvature $R$ is given by

$$
R=\left(H_{\alpha}^{\alpha}\right)^{2}-H_{\alpha}^{\beta} H_{\beta}^{\alpha} .
$$


In 2-dimensions $\int R \sqrt{g} d^{2} \xi$ is a topological invariant. However either of the two terms in (14) being separately reparametrization invariant can be used as a Lagrangian density for extrinsic curvature effects [1], modulo Euler characteristic.

Let us take $\left(\xi^{1}, \xi^{2}\right)$ to be the isothermal coordinates on $S$. We shall regard $S$ as a Riemann surface. Introducing complex coordinates $z=\xi^{1}+i \xi^{2}$ and $\bar{z}=\xi^{1}-i \xi^{2}$, the metric in a conformal gauge has the property

$$
\begin{aligned}
& g_{z z}=g_{\bar{z} \bar{z}}=0, \\
& g_{z \bar{z}}=g_{\bar{z} z} \neq 0 .
\end{aligned}
$$

(ii) The Grassmannian manifold $G_{2, n}$ is defined as a set of oriented 2-planes in $R^{n}$ passing through an origin. $G_{2, n}$ is isomorphic to $S O(n) / S O(2) \times S O(n-2)$. This may also be represented as a complex quadric $Q_{n-2}$ in $C P^{n-1}$ defined by

$$
\sum_{k=1}^{n} w_{k}^{2}=0
$$

where $w_{k} \in C$ are homogeneous coordinates of $C P^{n-1}$. To see this, we write $w_{k}=a_{k}+i b_{k}$, where $\left\{a_{k}\right\}$ and $\left\{b_{k}\right\}$ are real. (16) now reads as

$$
\begin{aligned}
\vec{A} \cdot \vec{B} & =0, \\
\|\vec{A}\| & =\|\vec{B}\|,
\end{aligned}
$$

where $\vec{A}=\left(a_{1}, \ldots ., a_{n}\right), \vec{B}=\left(b_{1}, \ldots ., b_{n}\right)$ and so $\vec{A}$ and $\vec{B}$ form an orthogonal basis for an oriented 2-plane in $R^{n}$.

(iii) The Gauss map $G: S \rightarrow G_{2, n} \simeq Q_{n-2}$ is defined by

$$
\partial_{z} X^{\mu}=\Psi \Phi^{\mu} ; \quad(\mu=1,2, \ldots \ldots n)
$$


where $\Phi^{\mu}$ is a point in $Q_{n-2}$ satisfying $\Phi^{\mu} \Phi^{\mu}=0$ (see Eqn.(16)) and $\Psi(\bar{z}, z)$ is yet to be determined. Note that this mapping makes sense as the induced metric $g_{\alpha \beta}=$ $\partial_{\alpha} X^{\mu} \partial_{\beta} X_{\mu}$, satisfies (15) (conformal immersion). Note further that every immersion in $R^{n}$ can be realized as a conformal immersion. While $\partial_{\bar{z}} \partial_{z} X^{\mu}$ is real, the term $\partial_{\bar{z}}\left(\Psi \Phi^{\mu}\right)$ may not in general be real. This together with the fact that $\partial_{\bar{z}} \partial_{z} X^{\mu}$ is normal to $S$ (see Eqn.12) determine $\Psi$ and the integrability conditions. From (12) using the notation $H^{\mu} \equiv H_{\alpha}^{\mu, \alpha} \equiv H_{\alpha}^{i \alpha} N_{i}^{\mu},(\mathrm{i}=1,2, \ldots . \mathrm{n}-2)$,

$$
H^{\mu}=\frac{2}{\lambda^{2}} \partial_{\bar{z}} \partial_{z} X^{\mu}
$$

where,

$$
\lambda^{2}=2\left\|\partial_{z} X^{\mu}\right\|^{2}=2|\Psi|^{2}\|\Phi\|^{2} .
$$

Expressing $H^{\mu}$ in terms of $\Psi, \Phi^{\mu}$ and noting that $H^{\mu}$ is normal to the surface while $\Phi^{\mu}$ is tangential, we obtain

$$
(\ln \Psi)_{\bar{z}}=-\eta
$$

and

$$
\begin{aligned}
V^{\mu} & =\partial_{\bar{z}} \Phi^{\mu}-\eta \Phi^{\mu} \\
& =\bar{\Psi}\|\Phi\|^{2} H^{\mu}
\end{aligned}
$$

where,

$$
\eta=\left(\partial_{\bar{z}} \Phi^{\mu}\right) \bar{\Phi}^{\mu} /\|\Phi\|^{2}
$$

(21) and (22) together contain (n-2) integrability conditions $[2,3,4]$ on the Gauss map $\Phi^{\mu}$. What it means is that not every $G_{2, n}$ field $\left(\Phi^{\mu}\right)$ forms a tangent plane to a given 
surface. We notice that the n-dimensional space-time emerges from the 2-dimensional $G_{2, n} \sigma$-model, through the reconstruction of surfaces from the $C P^{1}$ fields by

$$
X^{\mu}(z, \bar{z})=\int^{z} \operatorname{Re}\left\{\psi \Phi^{\mu} d z\right\}
$$

where the integrals are path independent [4].

(iv) Let us work out the details of Gauss map in $R^{3}$. $\Phi^{\mu}$ may be expressed in terms of a single complex function $F(\bar{z}, z)$ which is the inhomogeneous coordinate for $C P^{1}[2,4]$.

$$
\Phi^{\mu}=\left(1-F^{2}, i\left(1+F^{2}\right), 2 F\right)
$$

The normal Gauss map $N^{\mu}$ is given by

$$
N^{\mu}=\frac{1}{\left(1+|F|^{2}\right)}\left(F+\bar{F},-i(F-\bar{F}),|F|^{2}-1\right) .
$$

The Gauss map integrability condition is

$$
\operatorname{Im} \partial_{\bar{z}}\left(\frac{\partial_{z} \partial_{\bar{z}} F}{\partial_{\bar{z}} F}-2 \frac{\bar{F} \partial_{z} F}{1+|F|^{2}}\right)=0
$$

This is a third order differential constraint. A straightforward computation yields

$$
V^{\mu}=-2\left(\partial_{\bar{z}} F\right) N^{\mu}
$$

which allows us to write $\Psi$ in the form,

$$
\Psi(\bar{z}, z)=-\frac{\partial_{z} \bar{F}}{h\left(1+|F|^{2}\right)^{2}},
$$

where $h=H^{\mu} N^{\mu}$ is the scalar mean curvature.

(v) Let us introduce a local orthonormal moving frame $\hat{e}_{1}, \hat{e}_{2}, \hat{e}_{3} \equiv N^{\mu}$, where $\hat{e}_{1}$ and $\hat{e}_{2}$ are tangential to $S$. A convenient choice for $\hat{e}_{i}$ in terms of its image in the Gauss map is as follows [5];

$$
\hat{e}_{1}=\frac{1}{\left(1+|F|^{2}\right)}\left(1-\frac{F^{2}+\bar{F}^{2}}{2}, \frac{i}{2}\left(F^{2}-\bar{F}^{2}\right), F+\bar{F}\right),
$$




$$
\begin{gathered}
\hat{e}_{2}=\frac{1}{1+|F|^{2}}\left(\frac{i}{2}\left(F^{2}-\bar{F}^{2}\right), 1+\frac{F^{2}+\bar{F}^{2}}{2},-i(F-\bar{F})\right) \\
\hat{e}_{3}=\frac{1}{1+|F|^{2}}\left(F+\bar{F},-i(F-\bar{F}),|F|^{2}-1\right) .
\end{gathered}
$$

The structure equations (12) and (13) may be written in the following form:

$$
\begin{aligned}
\partial_{z} \hat{e}_{i} & =\left(A_{z}\right)_{i j} \hat{e}_{j}, \\
\partial_{\bar{z}} \hat{e}_{i} & =\left(A_{\bar{z}}\right)_{i j} \hat{e}_{j} .
\end{aligned}
$$

Using (29) to (31) one can express $A_{z}$ and $A_{\bar{z}}$ in terms of $F(\bar{z}, z)$ and its derivatives. From $\left(A_{z}\right)_{i j}=\hat{e}_{j} \partial_{z} \hat{e}_{i}$, we note that $\left(A_{z}\right)_{i j},\left(A_{\bar{z}}\right)_{i j}$ are antisymmetric. Under a local gauge transformation by $\mathcal{G} \in S O(3, C)$

$$
\hat{e}_{i} \rightarrow \mathcal{G}_{i j} \hat{e}_{j}
$$

a given surface $S$ is mapped into another surface $S^{\mathcal{G}}$. The vector potential $A_{\mu}$ then transforms as a gauge field. Note further that $\hat{e}_{1}$ and $\hat{e}_{2}$ are determined only upto an $S O(2, C)$ rotation. In the basis $(29)$ to $(31),\left(A_{z}\right)_{12}$ is non-vanishing. However, it was shown in [5] that by rotating $\hat{e}_{1}, \hat{e}_{2}$ through an angle $\alpha$ given by

$$
\alpha=\frac{-i}{2} \ln \left(\bar{\Psi}^{2}\|\Phi\|^{2}\right)
$$

where $\Psi$ and $\Phi^{\mu}$ were defined in (28), $A_{z} \rightarrow A^{\prime}{ }_{z}$ such that $\left(A^{\prime}{ }_{z}\right)_{12}=0$. A straightforward computation of the transformed gauge potentials yields the following (we drop the prime on $A_{z}$ and $A_{\bar{z}}$ from now on),

$$
A_{z}=\left(\begin{array}{ccc}
0 & 0 & \frac{1}{\sqrt{ } 2}\left(\frac{H_{z z}}{\sqrt{ } g}+h \sqrt{ } g\right) \\
0 & 0 & \frac{i}{\sqrt{ } 2}\left(-\frac{H_{z z}}{\sqrt{ } g}+h \sqrt{ } g\right) \\
-\frac{1}{\sqrt{ } g}\left(\frac{H_{z z}}{\sqrt{ } g}+h \sqrt{ } g\right) & -\frac{i}{\sqrt{ } 2}\left(-\frac{H_{z z}}{\sqrt{ } g}+h \sqrt{ } g\right) & 0
\end{array}\right),
$$


and

$$
A_{\bar{z}}=\left(\begin{array}{ccc}
0 & -\left[\partial_{\bar{z}}(\ln \bar{\Psi})+\frac{2 F \partial_{\bar{z}} \bar{F}}{1+|F|^{2}}\right] & \frac{1}{\sqrt{ } 2}\left(H_{\bar{z} \bar{z}}+h\right) \\
i\left[\partial_{\bar{z}} \ell n \bar{\Psi}+\frac{2 F \partial_{\bar{z}} \bar{F}}{1+|F|^{2}}\right] & 0 & \frac{i}{\sqrt{ } 2}\left(H_{\bar{z} \bar{z}}-h\right) \\
-\frac{1}{\sqrt{ } 2}\left(H_{\bar{z} \bar{z}}+h\right) & -\frac{i}{\sqrt{ } 2}\left(H_{\bar{z} \bar{z}}-h\right) & 0
\end{array}\right)
$$

In deriving (36) and (37) we have substituted back wherever possible, in terms of the characteristics of the 2-d surface from the Gauss map.

We now restrict to surfaces for which $h \sqrt{ } g=1$ and investigate the residual gauge degrees of freedom for $A_{z}$ which leaves it in the form (36) with only $H_{z z} / \sqrt{ } g$ as unfixed. This residual symmetry is a diffeomorphism. Among the three (complex) parameters determining $S O(3, C)$, two are determined in terms of the third, by the restrictions $A_{z}^{0}=$ $0, A_{z}^{-}=1$ ( here we are using the notation $A_{z}=A_{z}^{a} T^{a}, a=0, \pm$, and $T^{ \pm}$are the raising and lowering operators and $T^{0}$ is the Cartan generator for $\left.S O(3)\right)$. If we compute the transformation properties of the remaining degree of freedom in $A_{z}^{a}$, namely, $H_{z z} / \sqrt{ } g$, we find

$$
\delta_{\epsilon^{-}(\bar{z}, z)}\left(H_{z z} / \sqrt{ } g\right)=\partial_{z}^{3} \epsilon^{-}+2\left(\partial_{z} \epsilon^{-}\right)\left(\frac{H_{z z}}{\sqrt{ } g}\right)+\epsilon^{-} \partial_{z}\left(\frac{H_{z z}}{\sqrt{ } g}\right) .
$$

(38) is recognizable as the standard Virasaro action on energy momentum tensor. Similarly, looking at the transformation of $A_{\bar{z}} \equiv H_{\bar{z} \bar{z}}$, we find

$$
\delta_{\epsilon^{-}(\bar{z}, z)} H_{\bar{z} \bar{z}}=\partial_{\bar{z}} \epsilon^{-}+\epsilon^{-} \partial_{z} H_{\bar{z} \bar{z}}-\left(\partial_{z} \epsilon^{-}\right) H_{\bar{z} \bar{z}}
$$

We thus deduce that $H_{z z} / \sqrt{ } g$ transforms like energy-momentum tensor while $H_{\bar{z} \bar{z}}$ transforms like the metric tensor of a 2-d conformal field theory. We conclude that surfaces conformally immersed in $R^{3}$ with $h \sqrt{ } g=1$, exhibit Virasaro symmetry. This mechanism of generating diffeomorphism from gauge symmetry was first considered by Polyakov [10] in a different context. 
The consistency condition for (32) and (33) is found to be

$$
\partial_{\bar{z}} A_{z}-\partial_{z} A_{\bar{z}}+\left[A_{z}, A_{\bar{z}}\right] \equiv F_{\bar{z} z}=0
$$

(40) is identically satisfied if $g_{\alpha \beta}$ and $H_{\alpha \beta}$ are constructed from $X^{\mu}(\bar{z}, z)$ as in (11) and (12). We will take the point of view that $H_{z z} / \sqrt{ } g$ and $H_{\bar{z} \bar{z}}$ may be taken as independent dynamical degrees of freedom, independent of $X^{\mu}$ variables. Then the integrability condition (40) which can be rewritten as

$$
\partial_{z}^{3} H_{\bar{z} \bar{z}}=\left\{\partial_{\bar{z}}-H_{\bar{z} \bar{z}} \partial_{z}-2\left(\partial_{z} H_{\bar{z} \bar{z}}\right)\right\}\left(\frac{H_{z z}}{\sqrt{ } g}\right)
$$

will be taken as the equation of motion on $h \sqrt{ } g=1$ surfaces in which $H_{z z} / \sqrt{ } g$ and $H_{\bar{z} \bar{z}}$ will be treated as independent degrees of freedom.

(vi) We derive useful parametrizations for $H_{\bar{z} \bar{z}}$ and $H_{z z} / \sqrt{ } g$ in terms of the Gauss map of $h \sqrt{ } g=1$ surfaces. These parametrizations are used extensively in the next section. From (18), (24) and (28) we find that when $h \sqrt{ } g=1$

$$
\Psi \partial_{\bar{z}} F=-\frac{1}{2}
$$

From (12) it follows that $H_{\alpha \beta}=N^{\mu} \partial_{\alpha} \partial_{\beta} X^{\mu}$. Using the Gauss map, we find that $H_{\bar{z} \bar{z}}=-2 \bar{\Psi} \partial_{\bar{z}} \bar{F}$. Combining this with (42) we find

$$
H_{\bar{z} \bar{z}}=\frac{\partial_{\bar{z}} \bar{F}}{\partial_{z} \bar{F}} .
$$

For $h \sqrt{g}=1$ surfaces it can be verified that the integrability condition (26) is replaced by a stronger condition. Eliminating $\Psi$ from $(21)$ and $(42)$, we find that $F$ satisfies

$$
\frac{\partial_{\bar{z}} \partial_{\bar{z}} F}{\partial_{\bar{z}} F}-\frac{2 \bar{F} \partial_{\bar{z}} F}{1+|F|^{2}}=0 .
$$


Using this, a straightforward computation yields the following useful result for $H_{z z} / \sqrt{ } g$ when $h \sqrt{ } g=1$;

$$
\begin{aligned}
\frac{H_{z z}}{\sqrt{ } g} & =\frac{\partial_{z}^{3} \bar{F}}{\partial_{z} \bar{F}}-\frac{3}{2}\left(\frac{\partial_{z}^{2} \bar{F}}{\partial_{z} \bar{F}}\right)^{2} \\
& \equiv D_{z} \bar{F}
\end{aligned}
$$

In deriving (43) and (45) in terms of their images in the Grassmannian $G_{2,3}$, we have made use of the fact that both $H_{\bar{z} \bar{z}}$ and $H_{z z} / \sqrt{ } g$ are determined by the immersion coordinate $X^{\mu}$ of the surface $S$. In view of our remarks in (v) we take these as independent dynamical fields and thus parametrize them in terms of independent Gauss maps $F_{1}, F_{2}$ as

$$
H_{\bar{z} \bar{z}}=\frac{\partial_{\bar{z}} F_{2}}{\partial_{z} F_{2}}
$$

and

$$
\frac{H_{z z}}{\sqrt{ } g}=D_{z} F_{1}
$$

We shall look for an effective action which depends on $F_{1}$ and $F_{2}$ related to $H_{z z} / \sqrt{g}$ and $H_{\bar{z} \bar{z}}$ through (46) and (47). We shall use the equation of motion (41) to constrain these fields.

\section{INDUCED EXTRINSIC GRAVITY ACTION.}

In Section II, we showed that $h \sqrt{ } g=1$ surfaces exhibit Virasaro symmetry. We derive an action on such surfaces that is invariant under Virasaro transformations. The procedure used is as follows. We couple 2-d fermions in the vector representation of $S O(3)$ to the gauge fields $A_{z}, A_{\bar{z}}$ discussed in the previous section It is well known that upon integrating out the fermions, one gets the WZNW action [9]. We next evaluate this action

explicitly in terms of $F_{1}$ and $F_{2}$ (see Equations 46,47 ) for the partially gauge fixed $A_{z}$ 
given in (36), i.e., on $h \sqrt{ } g=1$ surfaces. Such an action was derived earlier in Ref.10 in a different context. For this reason, we shall be brief in our adaptation to $h \sqrt{ } g=1$ surfaces.

The gauge invariant action $\Gamma_{\text {eff }}$ depends on $A_{z}$ and $A_{\bar{z}}$ through the parametrizations $A_{z}=h^{-1} \partial_{z} h$ and $A_{\bar{z}}=g^{-1} \partial_{\bar{z}} g ; h, g \in S O(3)$. ( $h$ and $g$ here are not to be confused with mean curvature scalar and determinant of the induced metric). Note that choosing $h$ and $g$ as independent elements of the gauge group is consistent with taking $H_{\bar{z} \bar{z}}$ and $H_{z z} / \sqrt{ } g$ as independent of $X^{\mu}$. Explicitly

$$
\Gamma_{e f f}=\Gamma_{-}\left(A_{z}\right)+\Gamma_{+}\left(A_{\bar{z}}\right)-\frac{k}{4 \pi} \operatorname{Tr} \int A_{z} A_{\bar{z}} d z \wedge d \bar{z},
$$

where $k=n_{f}$ is the number of fermions and

$$
\begin{aligned}
\Gamma_{-}\left(A_{z}\right) & =\frac{k}{8 \pi} \operatorname{Tr} \int\left[\left(\partial_{z} h\right) h^{-1}\left(\partial_{\bar{z}} h\right) h^{-1}\right] d^{2} \xi \\
& +\frac{k}{12 \pi} \operatorname{Tr} \int \varepsilon^{a b c}\left(\left(\partial_{a} h\right) h^{-1}\left(\partial_{b} h\right) h^{-1}\left(\partial_{c} h\right) h^{-1}\right) d^{2} \xi d t,
\end{aligned}
$$

and $\Gamma_{+}\left(A_{\bar{z}}\right)$ is given by an expression similar to (49) with $h$ replaced by $g$ and the sign of the WZ term changed. Gauge invariance of $\Gamma_{e f f}$ is ensured by the last term in (48).

Next we apply the gauge restriction to (49).

$$
A_{z}^{-} \equiv h \sqrt{ } g=1 \quad ; \quad A_{z}^{0}=0 .
$$

A straightforward calculation yields

$$
\begin{aligned}
\Gamma_{-}\left(A_{z}^{-}=1, A_{z}^{0}=0, A_{z}^{+}=H_{z z} / \sqrt{ } g\right) \equiv & S_{-}\left(F_{1}\right)=\frac{k}{8 \pi} \int\left(\frac{\partial_{\bar{z}} F_{1}}{\partial_{z} F_{1}}\right) \\
& \left(\frac{\partial_{z}^{3} F_{1}}{\partial_{z} F_{1}}-2\left(\frac{\partial_{z}^{2} F_{1}}{\partial_{z} F_{1}}\right)^{2}\right) d z \wedge d \bar{z},
\end{aligned}
$$


where we have used (47) to write $H_{z z} / \sqrt{ } g$ in terms of $F_{1}$. (51) is the geometric action for the Virasaro group studied in [11]. It is not so easy to find $\Gamma_{+}\left(A_{\bar{z}}\right)$, when $A_{\bar{z}}$ is given by (37). However, we can evaluate it explicitly in a light-cone like gauge. Let us define the quantum action $S_{+}\left(H_{\bar{z} \bar{z}}\right)$ by

$$
\exp \left(-S_{+}\left(H_{\bar{z} \bar{z}}\right)\right)=\int\left[d A_{\bar{z}}\right] \delta\left(A_{\bar{z}}^{-}-H_{\bar{z} \bar{z}}\right) \exp \left(-\left(\Gamma_{+}\left(A_{\bar{z}}\right)-\int A_{\bar{z}}^{+}\right)\right)
$$

Evaluating (52) at the stationary path

$$
\frac{\delta \Gamma_{+}}{\delta A_{\bar{z}}^{0}}=J_{z}^{0}=0 \quad ; \quad \frac{\delta \Gamma_{+}}{\delta A_{\bar{z}}^{+}}=J_{z}^{-}=1,
$$

we obtain the classical limit which can be explicitly evaluated [10] as (Note that $H_{\bar{z} \bar{z}}$ is the component $A_{\bar{z}}^{-}$in $\left.(37)\right)$

$$
S_{+}\left(F_{2}\right)=-\frac{k}{8 \pi} \int \frac{\partial_{z}^{2} F_{2}}{\partial_{z} F_{2}}\left(\frac{\partial_{z} \partial_{\bar{z}} F_{2}}{\partial_{z} F_{2}}-\frac{\partial_{z}^{2} F_{2}}{\partial_{z} F_{2}} \cdot \frac{\partial_{\bar{z}} F_{2}}{\partial_{z} F_{2}}\right) d z \wedge d \bar{z} .
$$

(54) is the gauge fixed form of $\Gamma_{+}\left(A_{\bar{z}}\right)$ in the gauge determined by (53). Note that it is analogous to the light-cone gauge fixing in 2-d intrinsic gravity theory. (54) is precisely of the form of the light-cone action in 2-d intrinsic gravity theory.

The variations of $S_{-}\left(F_{1}\right)$ and $S_{+}\left(F_{2}\right)$ under infinitesimal changes (in $\left.F_{1}, F_{2}\right)$ are given by

$$
\delta S_{-}=-\frac{k}{4 \pi} \int\left(\delta_{\epsilon} F_{1}\right) \frac{\partial_{\bar{z}}\left(D_{z} F_{1}\right)}{\partial_{z} F_{1}} d z \wedge d \bar{z}
$$

and

$$
\delta S_{+}=-\frac{k}{4 \pi} \int\left(\delta_{\epsilon} F_{2}\right) \frac{1}{\partial_{z} F_{2}} \cdot \partial_{z}^{3}\left(\frac{\partial_{\bar{z}} F_{2}}{\partial_{z} F_{2}}\right) d z \wedge d \bar{z}
$$

The third term on the right hand side of (48) in the gauge $A_{z}^{0}=0, A_{z}^{-}=1$ becomes $\int\left(A_{z}^{+} A_{\bar{z}}^{-}+A_{\bar{z}}^{+}\right) d^{2} \xi$, of which the second term has been taken into account in (52). The 
remaining term, i.e., $\int A_{z}^{+} A_{\bar{z}}^{-}$, with the identification $A_{z}^{+}=H_{z z} / \sqrt{ } g=D_{z} F_{1}$ and $A_{\bar{z}}^{-}=H_{\bar{z} \bar{z}}=\partial_{\bar{z}} F_{2} / \partial_{z} F_{2}$ yields $\frac{k}{4 \pi} \int\left(D_{z} F_{1}\right)\left(\frac{\partial_{\bar{z}} F_{2}}{\partial_{z} F_{2}}\right)$ and so the total action on $h \sqrt{ } g=1$ surfaces reads

$$
\begin{aligned}
\Gamma_{e f f}\left(F_{1}, F_{2}\right) & =\frac{k}{8 \pi} \int \frac{\partial_{\bar{z}} F_{1}}{\partial_{z} F_{1}}\left(\frac{\partial_{z}^{3} F_{1}}{\partial_{z} F_{1}}-2\left(\frac{\partial_{z}^{2} F_{1}}{\partial_{z} F_{1}}\right)^{2}\right) d z \wedge d \bar{z} \\
& -\frac{k}{8 \pi} \int \frac{\partial_{z}^{2} F_{2}}{\partial_{z} F_{2}}\left(\frac{\partial_{z} \partial_{\bar{z}} F_{2}}{\partial_{z} F_{2}}-\frac{\partial_{z}^{2} F_{2}}{\partial_{z} F_{2}} \cdot \frac{\partial_{\bar{z}} F_{2}}{\partial_{z} F_{2}}\right) d z \wedge d \bar{z} \\
& -\frac{k}{4 \pi} \int \frac{\partial_{\bar{z}} F_{2}}{\partial_{z} F_{2}} D_{z} F_{1} d z \wedge d \bar{z}
\end{aligned}
$$

Equation (57) is our main result in this section We have derived the extrinsic geometric gravitational WZNW action on $h \sqrt{ } g=1$ surfaces in the light-cone like gauge (53). The induced extrinsic 2-d gravity action combines in a gauge invariant way the geometric and light-cone actions which have been studied in the context of 2 -d intrinsic gravity. It is remarkable that a gauge invariant interacting theory of these two actions has physical implication in the theory on $h \sqrt{ } g=1$ surfaces.

Note that restricted gauge transformations have yielded diffeomorphism due to which $A_{z}^{+}$which initially had conformal spin 1 , has become $H_{z z} / \sqrt{ } g=D_{z} F_{1}$, a conformal spin 2 object. Same remark applies to $A_{\bar{z}}^{-} \rightarrow \frac{\partial_{\bar{z}} F_{2}}{\partial_{z} F_{2}}$, which acquires a conformal spin 2 . This suggests that $F_{2}$ should be regarded as a primary conformal field with conformal weight 0 in $\bar{z}$ sector. Later, we show that quantum corrections modify this property of $F_{2}$.

\section{SYMMETRY PROPERTIES OF $\Gamma_{e f f}$.}

In this section we discuss the symmetry properties of the gauge fixed action (57) on $h \sqrt{ } g=1$ surfaces in $R^{3}$. The equation of motion for $(57)$ is

$$
\partial_{z}^{3}\left(\frac{\partial_{\bar{z}} F_{2}}{\partial_{z} F_{2}}\right)-\partial_{\bar{z}} D_{z} F_{1}-\left(\frac{\partial_{\bar{z}} F_{2}}{\partial_{z} F_{2}}\right) \partial_{z} D_{z} F_{1}-2 \partial_{z}\left(\frac{\partial_{\bar{z}} F_{2}}{\partial_{z} F_{2}}\right) D_{z} F_{1}=0
$$


Using (46) and (47), it is readily seen that (58) is equivalent to (8) which we referred to as the anomaly equation. It should be mentioned here that varying (57) with respect to $F_{1}$ and $F_{2}$ independently yielded the single equation of motion (58). Our objective is to construct an effective action in terms of $H_{\bar{z} \bar{z}}$ and $H_{z z} / \sqrt{ } g$ through their parameterization (46) and (47) such that these fields are independent of $X^{\mu}$.

Let us first verify that (57) is invariant under infinitesimal Virasaro transformations (38) and (39). It can be easily verified that these transformations for $H_{\bar{z} \bar{z}}$ and $H_{z z} / \sqrt{ } g$ are equivalent to the following transformation for $F_{i}(\mathrm{i}=1,2)$.

$$
\delta_{\epsilon^{-}} F_{i}=\epsilon^{-}(\bar{z}, z) \partial_{z} F_{i}, \quad(i=1,2)
$$

Invariance of (57) under (59) may be verified readily using (55) and (56). Now (59) is the infinitesimal version of the finite transformations;

$$
\begin{aligned}
& F_{1}(\bar{z}, z) \rightarrow F_{1}(\bar{z}, f(\bar{z}, z)) \equiv F_{1} \circ f \\
& F_{2}(\bar{z}, z) \rightarrow F_{2}(\bar{z}, f(\bar{z}, z)) \equiv F_{2} \circ f .
\end{aligned}
$$

The infinitesimal version (59) corresponds to

$$
f(\bar{z}, z)=z+\epsilon^{-}(\bar{z}, z) .
$$

We therefore conclude that

$$
\Gamma_{e f f}\left(F_{1}, F_{2}\right)=\Gamma_{e f f}\left(F_{1} \circ f, F_{2} \circ f\right) .
$$

Eqn.(62) has an interesting consequence. If we choose $f=F_{2}^{-1}(\bar{z}, z)$, where the inverse function is defined by the relation

$$
F_{2}\left(\bar{z}, F_{2}^{-1}(\bar{z}, z)\right)=z
$$


we find that

$$
\Gamma_{e f f}\left(F_{1}, F_{2}\right)=\Gamma_{-}\left(F_{1} \circ F_{2}^{-1}\right)=\Gamma_{+}\left(F_{2} \circ F_{1}^{-1}\right)
$$

where the last equality follows by the interchange, $F_{1} \leftrightarrow F_{2}$. We thus have derived the following useful composition formula.

$$
\begin{aligned}
\Gamma_{+}\left(F_{2} \circ F_{1}^{-1}\right) & =\Gamma_{-}\left(F_{1} \circ F_{2}^{-1}\right) \\
& =\Gamma_{+}\left(F_{2}\right)+\Gamma_{-}\left(F_{1}\right)-\frac{k}{4 \pi} \int \frac{\partial_{\bar{z}} F_{2}}{\partial_{z} F_{2}} D_{z} F_{1}
\end{aligned}
$$

From (64) we conclude that the properties of the induced extrinsic gravity action can be understood from those of either the light-cone action $\Gamma_{+}\left(F_{2} \circ F_{1}^{-1}\right)$ encountered in 2-d (intrinsic) gravity theory with the replacement of $f$ of intrinsic 2-d gravity theory by $F_{2} \circ F_{1}^{-1}$ or from those of $\Gamma_{-}\left(F_{1} \circ F_{2}^{-1}\right)$ i.e., the geometric action which arises in the quantization of the Virasaro group by coadjoint orbits (with the replacement of $f$ by $\left.F_{1} \circ F_{2}^{-1}\right)$.

The equation of motion for the light-cone action for $F_{2} \circ F_{1}^{-1}$ is [7]

$$
\partial_{z}^{3}\left(\frac{\partial_{\bar{z}}\left(F_{2} \circ F_{1}^{-1}\right)}{\partial_{z}\left(F_{2} \circ F_{1}^{-1}\right)}\right)=0 .
$$

By using the rules for derivatives of the composed function $F_{2} \circ F_{1}^{-1}$ with respect to $z$ and $\bar{z}$, in terms of those of $F_{1}$ and $F_{2}$, it is readily verified that (66) reduces to the equation of motion (58). From (66), we conclude that $\Gamma_{\text {eff }}\left(F_{1}, F_{2}\right)$ has a hidden $S L(2, C)$ [at the algebra level it is same as $S L(2, R)$ ] symmetry in the $\bar{z}$-sector as in the intrinsic 2-d gravity theory. Recall that the $S L(2, C)$ currents $J_{\bar{z}}^{a}(a= \pm, 0)$ are defined through

$$
\frac{\partial_{\bar{z}}\left(F_{2} \circ F_{1}^{-1}\right)}{\partial_{z}\left(F_{2} \circ F_{1}^{-1}\right)}=J_{\bar{z}}^{(+)}(\bar{z})-2 z J_{\bar{z}}^{0}+z^{2} J_{\bar{z}}^{-}(\bar{z})
$$


where the currents are functions of $\bar{z}$ only. A straightforward calculation yields the following expressions for the currents.

$$
\begin{aligned}
J_{\bar{z}}^{+} & =-\frac{k}{2}\left[\left(\partial_{z} F_{1}\right)\left(\frac{\partial_{\bar{z}} F_{2}}{\partial_{z} F_{2}}\right)-\partial_{\bar{z}} F_{1}\right] \\
& -2 F_{1} J_{\bar{z}}^{0}+F_{1}^{2} J_{\bar{z}}^{-}, \\
J_{\bar{z}}^{0}= & -\frac{k}{2} F_{1} J_{\bar{z}}^{-}+\frac{k}{2}\left[\frac{\partial_{z} \partial_{\bar{z}} F_{2}}{\partial_{z} F_{2}}-\left(\frac{\partial_{\bar{z}} F_{2}}{\partial_{z} F_{2}}\right) \frac{\partial_{z}^{2} F_{2}}{\partial_{z} F_{2}}\right. \\
- & \left.\frac{\partial_{z} \partial_{\bar{z}} F_{1}}{\partial_{z} F_{1}}+\frac{\partial_{\bar{z}} F_{2}}{\partial_{z} F_{2}} \cdot \frac{\partial_{z}^{2} F_{1}}{\partial_{z} F_{1}}\right],
\end{aligned}
$$

and

$$
\begin{aligned}
J_{\bar{z}}^{-} & =-\frac{k}{2}\left(\frac{\partial_{z}^{2} F_{1} \cdot \partial_{z} \partial_{\bar{z}} F_{1}}{\left(\partial_{z} F_{1}\right)^{3}}-\frac{\partial_{\bar{z}} \partial_{z}^{2} F_{1}}{\left(\partial_{z} F_{1}\right)^{2}}\right) \\
& --\frac{k}{2\left(\partial_{z} F_{1}\right)}\left[\partial_{z}^{2}\left(\frac{\partial_{\bar{z}} F_{2}}{\partial_{z} F_{2}}\right)+\left(\frac{\partial_{z}^{2} F_{1}}{\partial_{z} F_{1}}\right) \partial_{z}\left(\frac{\partial_{\bar{z}} F_{2}}{\partial_{z} F_{2}}\right)\right. \\
& \left.+\frac{\partial_{\bar{z}} F_{2}}{\partial_{z} F_{2}}\left[\frac{\partial_{z}^{3} F_{1}}{\partial_{z} F_{1}}-\frac{\left(\partial_{z}^{2} F_{1}\right)^{2}}{\left(\partial_{z} F_{1}\right)^{2}}\right]\right] .
\end{aligned}
$$

It is straightforward to verify that

$$
\partial_{z} J_{\bar{z}}^{a}=0 .
$$

In order to exhibit $S L(2, C)$ invariance of the action (57), it is convenient to identify it with the geometric action for $F_{1} \circ F_{2}^{-1}$. Recall that [11] the geometric action is invariant under $S L(2, C)$ transformations:

$$
F_{1} \circ F_{2}^{-1} \stackrel{S L(2, C)}{\rightarrow} \frac{a(\bar{z}) F_{1} \circ F_{2}^{-1}+b(\bar{z})}{c(\bar{z}) F_{1} \circ F_{2}^{-1}+d(\bar{z})},
$$

with $a d-b c=1$. Noether currents for these transformations, whose infinitesimal form we write as

$$
\delta_{S L(2, C)} F_{1} \circ F_{2}^{-1}=\epsilon_{-}(\bar{z})+\epsilon_{0}(\bar{z}) F_{1} \circ F_{2}^{-1}+\epsilon_{+}(\bar{z})\left(F_{1} \circ F_{2}^{-1}\right)^{2},
$$


are precisely those given in (68) to $(70)$.

Next, we discuss the consequences of reparametrization invariance of (57). In the $\bar{z}$-sector, an infinitesimal conformal transformation results in

$$
\delta_{\bar{\epsilon}} F_{i}=\bar{\epsilon}(\bar{z}) \partial_{\bar{z}} F_{i} ; \quad(i=1,2)
$$

Expressing the change in $\Gamma_{\text {eff }}$ as

$$
\delta_{\bar{\epsilon}} \Gamma_{e f f}\left(F_{1}, F_{2}\right)=-\frac{1}{2 \pi} \int d z \wedge d \bar{z} \bar{\epsilon}(\bar{z}) \partial_{z} T_{\bar{z} \bar{z}}
$$

we find

$$
T_{\bar{z} \bar{z}}\left(F_{1}, F_{2}\right)=T_{\bar{z} \bar{z}}\left(F_{1}\right)+T_{\bar{z} \bar{z}}\left(F_{2}\right)+T_{\bar{z} \bar{z}}^{I n t}\left(F_{1}, F_{2}\right)
$$

where,

$$
\begin{gathered}
T_{\bar{z} \bar{z}}\left(F_{1}\right)=-\frac{k}{2}\left\{\frac{\left(\partial_{\bar{z}} F_{1}\right)\left(\partial_{z}^{2} \partial_{\bar{z}} F_{1}\right)}{\left(\partial_{z} F_{1}\right)^{2}}-\frac{1}{2}\left(\frac{\partial_{\bar{z}} \partial_{z} F_{1}}{\partial_{z} F_{1}}\right)^{2}-\frac{\partial_{z}^{2} F_{1} \partial_{\bar{z}} F_{1} \partial_{z} \partial_{\bar{z}} F_{1}}{\left(\partial_{z} F_{1}\right)^{2}}\right\} \\
T_{\bar{z} \bar{z}}\left(F_{2}\right)=\frac{k}{2}\left[\left(\partial_{z}\left(\frac{\partial_{\bar{z}} F_{2}}{\partial_{z} F_{2}}\right)\right)^{2}-2\left(\frac{\partial_{\bar{z}} F_{2}}{\partial_{z} F_{2}}\right) \partial_{z}^{2}\left(\frac{\partial_{\bar{z}} F_{2}}{\partial_{z} F_{2}}\right)\right]
\end{gathered}
$$

and

$$
\begin{aligned}
T_{\bar{z} \bar{z}}^{I n t}\left(F_{1}, F_{2}\right) & =\frac{k}{2}\left\{( \frac { \partial _ { \overline { z } } F _ { 1 } } { \partial _ { z } F _ { 1 } } ) \left[\partial_{z}\left(\frac{\partial_{\bar{z}} F_{2}}{\partial_{z} F_{2}}\right) \frac{\partial_{z}^{2} F_{1}}{\partial_{z} F_{1}}\right.\right. \\
& \left.+\left(\frac{\partial_{\bar{z}} F_{2}}{\partial_{z} F_{2}}\right)\left(\frac{\partial_{z}^{3} F_{1}}{\partial_{z} F_{1}}-\left(\frac{\partial_{z}^{2} F_{1}}{\partial_{z} F_{1}}\right)^{2}\right)+\partial_{z}^{2}\left(\frac{\partial_{\bar{z}} F_{2}}{\partial_{z} F_{2}}\right)\right] \\
& -\frac{\partial_{z} \partial_{\bar{z}} F_{1}}{\partial_{z} F_{1}}\left[2 \frac{\partial_{\bar{z}} F_{2}}{\partial_{z} F_{2}}\left(\frac{\partial_{z}^{2} F_{1}}{\partial_{z} F_{1}}\right)+\partial_{z}\left(\frac{\partial_{\bar{z}} F_{2}}{\partial_{z} F_{2}}\right)\right] \\
& \left.+\frac{\partial_{z}^{2} \partial_{\bar{z}} F_{1}}{\partial_{z} F_{1}}\left(\frac{\partial_{\bar{z}} F_{2}}{\partial_{z} F_{2}}\right)-\left(\frac{\partial_{\bar{z}} F_{2}}{\partial_{z} F_{2}}\right)^{2} D_{z} F_{1}\right\}
\end{aligned}
$$

(77) and (78) can be recognized to be the corresponding $T_{\bar{z} \bar{z}}$ for the geometric and lightcone actions respectively. 
It is readily verified that, using the equation of motion (58),

$$
\partial_{z} T_{\bar{z} \bar{z}}\left(F_{1}, F_{2}\right)=0
$$

We expect $T_{\bar{z} \bar{z}}\left(F_{1}, F_{2}\right)$ to be of Sugawara form, and indeed, it can be verified that

$$
T_{\bar{z} \bar{z}}\left(F_{1}, F_{2}\right)=-\frac{1}{k} \eta_{a b} J_{\bar{z}}^{a} J_{\bar{z}}^{b}
$$

where,

$$
\eta_{00}=1 ; \quad \eta_{1-1}=\eta_{-11}=-\frac{1}{2}
$$

We thus conclude that the induced 2-d extrinsic gravity theory on $h \sqrt{ } g=1$ surfaces in $R^{3}$ has conformal invariance in the $\bar{z}$-sector. Furthermore, this conformal field theory is in the same universality class as the induced 2-d intrinsic gravity theory.

Let us briefly discuss the energy-momentum tensor $T_{z z}\left(F_{1}, F_{2}\right)$. Under infinitesimal conformal change

$$
\delta_{\epsilon(z)} F_{i}(\bar{z}, z)=\epsilon(z) \partial_{z} F_{i}(\bar{z}, z) ; \quad(i=1,2)
$$

This leads to

$$
\delta_{\epsilon} \Gamma_{e f f}\left(F_{1}, F_{2}\right)=\frac{1}{2 \pi} \int \epsilon(z) \nabla_{\bar{z}}\left(F_{2}\right) T_{z z}
$$

where,

$$
\nabla_{\bar{z}}\left(F_{2}\right) T_{z z}=\partial_{\bar{z}} T_{z z}-\left(\frac{\partial_{\bar{z}} F_{2}}{\partial_{z} F_{2}}\right) \partial_{z} T_{z z}-2 \partial_{z}\left(\frac{\partial_{\bar{z}} F_{2}}{\partial_{z} F_{2}}\right) T_{z z}
$$

and

$$
T_{z z}\left(F_{1}, F_{2}\right)=\frac{k}{2}\left[D_{z} F_{1}-D_{z} F_{2}\right] .
$$


From the equation of motion, one finds that

$$
\nabla_{\bar{z}}\left(F_{2}\right) T_{z z}\left(F_{1}, F_{2}\right)=0
$$

The transformation of the $S L(2, C)$ currents and $T_{\bar{z} \bar{z}}\left(F_{1}, F_{2}\right)$ under conformal and $S L(2, C)$ transformations can be easily worked out. We give below the results for completeness and will be used in quantizing the theory in the next section

(i) $S L(2, C)$ Variations:

$$
\delta_{S L(2, C)} J_{\bar{z}}=-\frac{k}{2}\left(\partial_{\bar{z}} \epsilon\right)-\left[\epsilon, J_{\bar{z}}\right],
$$

where,

$$
\begin{gathered}
J_{\bar{z}}=\eta_{a b} T^{a} J_{\bar{z}}^{a}, \\
\epsilon=\epsilon_{a}(\bar{z}) T^{a}, \\
T^{0}=\frac{1}{2}\left[\begin{array}{cc}
-1 & 0 \\
0 & 1
\end{array}\right], T^{+}=\left[\begin{array}{ll}
0 & 0 \\
1 & 0
\end{array}\right], T^{-}=\left[\begin{array}{cc}
0 & -1 \\
0 & 0
\end{array}\right] \\
{\left[T^{a}, T^{b}\right]=f_{c}^{a b} T^{c},} \\
f_{0}^{1-1}=-2, f_{1}^{01}=-f_{-1}^{0-1}=1 .
\end{gathered}
$$

(ii) Conformal Variations:

$$
\delta_{\bar{\epsilon}(\bar{z})} J_{\bar{z}}^{a}=\bar{\epsilon}(\bar{z}) \partial_{z} J_{\bar{z}}^{a}+\left(\partial_{\bar{z}} \bar{\epsilon}\right) J_{\bar{z}}^{a}, \quad(a= \pm, 0),
$$


and

$$
\delta_{\bar{\epsilon}(\bar{z})} T_{\bar{z} \bar{z}}\left(F_{1}, F_{2}\right)=\bar{\epsilon}(\bar{z}) \partial_{\bar{z}} T_{\bar{z} \bar{z}}\left(F_{1}, F_{2}\right)+2\left(\partial_{\bar{z}} \bar{\epsilon}\right) T_{\bar{z} \bar{z}}\left(F_{1}, F_{2}\right)
$$

(88) and (94) follow from (68)-(70) and (80) using

$$
\delta_{\bar{\epsilon}(\bar{z})} F_{i}=\bar{\epsilon}(\bar{z}) \partial_{\bar{z}} F_{i}, \quad(i=1,2)
$$

Before quantizing the induced extrinsic gravity theory in the $\bar{z}$-sector, we must first modify the conformal properties of $F_{1}$. If we regard $\Gamma_{\text {eff }}$ in (57) as equivalent to the geometric action for $F_{1} \cdot F_{2}^{-1} \equiv F_{1}\left(\bar{z}, F_{2}^{-1}(\bar{z}, z)\right)$, then following Aoyoma [12], one should modify the conformal weight of $F_{1} \cdot F_{2}^{-1}$ in the $\bar{z}$-sector, such that

$$
\delta_{\bar{\epsilon}(\bar{z})}\left(F_{1} \cdot F_{2}^{-1}\right)=\bar{\epsilon}(\bar{z}) \partial_{\bar{z}}\left(F_{1} \cdot F_{2}^{-1}\right)+\left(\partial_{\bar{z}} \bar{\epsilon}\right)\left(F_{1} \cdot F_{2}^{-1}\right)
$$

(97) which assigns conformal spin 1 for $F_{1}$ in $\bar{z}$-sector, is necessitated by the equivalence of the geometric action with the Liouville theory.

The same conclusion can also be arrived at looking from the point of view of the light-cone action. Following [7], let us observe that (57) expressed as $\Gamma_{+}\left(F_{2} \cdot F_{1}^{-1}\right)$ is the gauge fixed form of the action on $h \sqrt{ } g=1$ surfaces, in the gauge, $\frac{\delta \Gamma_{+}}{\delta A_{\bar{z}}^{0}}=0, \frac{\delta \Gamma_{+}}{\delta A_{\bar{z}}^{+}}=1$ (See (53)). The quantum action $S_{+}\left(F_{2} \cdot F_{1}^{-1}\right)$ which guarantees general covariance will contain a term proportional to $\int\left(\frac{\delta \Gamma_{+}}{\delta A_{\bar{z}}}\right) T_{\bar{z} \bar{z}}$. This has been shown in [7] to yield an extra term to $T_{\bar{z} \bar{z}}$ which is $\partial_{\bar{z}} J_{\bar{z}}^{0}$. This is equivalent to the modification given in terms of $F_{1}$ and $F_{2}$ as

$$
\begin{aligned}
\delta_{\bar{\epsilon}(\bar{z})} F_{1} & =\bar{\epsilon}(\bar{z}) \partial_{\bar{z}} F_{1}+\left(\partial_{\bar{z}} \bar{\epsilon}\right) F_{1}, \\
\delta_{\bar{\epsilon}(\bar{z})} F_{2} & =\bar{\epsilon}(\bar{z}) \partial_{\bar{z}} F_{2} .
\end{aligned}
$$

Under this modification, (80) still holds with the modified energy-momentum tensor

$$
T_{\bar{z} \bar{z}}^{m o d}(\bar{z})=-\frac{1}{k} \eta^{a b} J_{\bar{z}}^{a} J_{\bar{z}}^{b}-\partial_{\bar{z}} J_{\bar{z}}^{0}
$$


Under the modified conformal transformations, the conformal weight of the currents also change. We find instead of (94)

$$
\delta_{\bar{\epsilon}(\bar{z})} J_{\bar{z}}^{a}=\left(\bar{\epsilon}(\bar{z}) \partial_{\bar{z}}+(1+a) \partial_{\bar{z}} \bar{\epsilon}(\bar{z})\right) J_{\bar{z}}^{a},
$$

while, (95) remains valid for $T_{\bar{z} \bar{z}}^{\bmod }\left(F_{1}, F_{2}\right)$.

\section{QUANTUM THEORY ON $h \sqrt{ } g=1$ SURFACES IMMERSED IN $R^{3}$}

We have all the machinery in place for quantizing our theory on $h \sqrt{ } g=1$ surfaces in $R^{3}$. The $\bar{z}$-sector of the action (57) can be quantized like the WZNW action [13]. The details of this procedure are hardly new and we are contend with merely summarizing the essential steps. From (68) to (70), we can expand $J_{\bar{z}}^{a}$ in Laurent series as

$$
J_{\bar{z}}^{a}=\sum_{n=-\infty}^{\infty} J_{n}^{a} \bar{z}^{-n-1-a}
$$

while from (99), $T_{\bar{z} \bar{z}}^{m o d}$ is expanded as

$$
T_{\bar{z} \bar{z}}^{\text {mod }}=\sum_{n=-\infty}^{\infty} L_{n} \bar{z}^{-n-2}
$$

Then, consistent with the $S L(2, C)$ transformations (88), the currents are quantized as [see also [14]]

$$
\left[J_{n}^{a}, J_{m}^{b}\right]=-\frac{k}{2}(n+a) \eta^{a b} \delta_{n+m, 0}+f_{c}^{a b} J_{n+m}^{c}
$$

with

$$
J_{n}^{a} \mid 0>=0
$$

for $n>-a$. Next, we define the quantum version of the energy-momentum tensor $T_{\bar{z} \bar{z}}$ or $L_{n}$. As shown in [13], it is given by

$$
L_{n}=\frac{1}{(k+2)} \sum_{m=-\infty}^{\infty} \eta_{a b}: J_{m}^{a} J_{n-m}^{b}:+(n+1) J_{n}^{0},
$$


where the normal ordering (: :) is understood as

$$
\sum_{m=-\infty}^{\infty}: J_{m}^{a} J_{n-m}^{b}:=\sum_{m=-\infty}^{(n+b)} J_{m}^{a} J_{n-m}^{b}+\sum_{(m=n+b+1)}^{\infty} J_{n-m}^{b} J_{m}^{a} .
$$

We are now in a position to calculate the rest of the commutators. We find [12]

$$
\left[L_{n}, J_{m}^{a}\right]=-(m-a n) J_{n+m}^{a}-\frac{k}{2} n(n+1) \eta^{a 0} \delta_{n+m}
$$

and

$$
\left[L_{n}, L_{m}\right]=(n-m) L_{n+m}+\frac{c_{L}}{12} n(n-1)(n+1) \delta_{n+m, 0}
$$

where,

$$
\begin{aligned}
c_{L} & =\frac{3 k}{k+2}-6 k, \\
& =15-6(k+2)-\frac{6}{k+2} .
\end{aligned}
$$

Note that $-6 k$ comes from the modified term in $T_{\bar{z} \bar{z}}$. Thus the central charge of the Virasaro algebra in the left sector on $h \sqrt{ } g=1$ surfaces is the same as for the 2-d intrinsic gravity in the same sector.

It is interesting to note that in Section II, we observed that conformally immersed surfaces in $R^{3}$ with $h \sqrt{ } g=1$ exhibit Virasaro invariance with $\frac{H_{z z}}{\sqrt{ } g} \equiv D_{z} F_{1}$ transforming like the energy-momentum tensor and yet, it is the $\bar{z}$-sector of this theory which can be successfully quantized. For the z-sector because of (87), the central charge remains unknown.

\section{VI.CONCLUSIONS}

In this article we have studied the classical and quantum properties of a class of conformally immersed surfaces in background $R^{3}$. The class of surfaces is characterized 
by their extrinsic geometric property; i.e., $h \sqrt{ } g=1$. We have constructed an action on such surfaces which depends on $\frac{H_{z z}}{\sqrt{ } g}$ and $H_{\bar{z} \bar{z}}$ as dynamical field variables. Here $H_{z z}$ and $H_{\bar{z} \bar{z}}$ are components of the second fundamental form of the surface and $g$ is the determinant of the induced metric on the surface. The construction of the extrinsic geometric WZNW action is done by minimally coupling 2-d fermions to gauge fields on the surface, constructed from the structure equations to the surface. We show that the gauge restriction $h \sqrt{ } g=1$ has an explicit representation which is equivalent to a gauge invariant coupling of light-cone and geometric type actions studied in the literature in connection with intrinsic 2-d gravity theory.

We emphasize in this article the importance of the role of the Gauss map in establishing the existence of Virasaro symmetry in $h \sqrt{ } g=1$ surfaces. The Gauss map of conformally immeresed surfaces in $R^{n}$, studied in detail elsewhere, is the map from a surface in the Grassmannian manifold $G_{2, n}$. Since, not every Grassmannian manifold field forms tangent planes to a surface in $R^{n},(n-2)$ integrability conditions must be satisfied by the $G_{2, n}$ fields. The effective action $\Gamma_{e f f}\left(F_{1}, F_{2}\right)$ is a function of two complex functions $F_{1}$, and $F_{2}$ which parameterize $H_{z z} / \sqrt{ } g=D_{z} F_{1}$, and $H_{\bar{z} \bar{z}}=\frac{\partial_{\bar{z}} F_{2}}{\partial_{z} F_{2}}$ and are shown to be the image of $X^{\mu}$ in $G_{2,3}$. The effective action $\Gamma_{\text {eff }}\left(F_{1}, F_{2}\right)=\Gamma_{-}\left(F_{1} \cdot F_{2}^{-1}\right)=\Gamma_{+}\left(F_{2} \cdot F_{1}^{-1}\right)$ is shown to be the Grassmannian $\sigma$-model action for induced extrinsic curvature action. Because of a useful composition formula for $\Gamma_{\text {eff }}\left(F_{1}, F_{2}\right)$, it is found that the induced 2-d extrinsic gravity theory in $R^{3}$ on $h \sqrt{ } g=1$ surfaces is in the same universality class as the intrinsic 2-d gravity theory. This result constitutes the main observation in this work. Quantization of $h \sqrt{ } g=1$ surfaces is carried out with the $S L(2, C)$ currents in the $\bar{z}$-sector of the theory.

\section{Acknowledgements}


We are thankful to I.Volovich and I.Y.Arefeva for useful discussions. This work has been supported by an operating grant from the Natural Sciences and Engineering Research Council of Canada. One of the authors (R.P) thanks the Department of Physics for the kind hospitality during his stay at Simon Fraser University where part of the work was done.

\section{References}

1. A.M.Polyakov, Nucl.Phys. B268 (1986) 406;

W.Helfrich, J.Phys.(Paris) 46 (1985) 1263;

L.Peliti and S.Leibler, Phys.Rev.Lett. 54 (1985) 1690.

2. K.S.Viswanathan, R.Parthasarathy and D.Kay, Ann.Phys.(N.Y) (1991) 237.

3. R.Parthasarathy and K.S.Viswanathan, Int.J.Mod.Phys. A7 (1992) 1819.

4. D.A.Hoffman and R.Osserman, Proc.London.Math.Soc.(3) 50 (1985) 21; J.Diff.Geom. 18 (1983) 733.

5. R.Parthasarathy and K.S.Viswanathan, Int.J.Mod.Phys. A7 (1992) 317.

6. G.M.Sotkov, M.Stanishkov and C.J.Zhu, Nucl.Phys. B356 (1991) 245.

7. A.M.Polyakov, Mod.Phys.Lett. A2 (1987) 893.

8. V.G.Knizhnik, A.M.Polyakov and A.B.Zamalodchikov, Mod.Phys. Lett. A3 (1988) 819. 
9. A.M.Polyakov, in Fields, Strings and Critical Phenomena Les Houches, 1988; Eds.E.Brezin and J.Zinn-Justin. North Holland (1990).

10. A.M.Polyakov, Int.J.Mod.Phys. A5 (1990) 833. (see also in Physics and Mathematics of Strings; Eds.L.Brink, D.Friedman and A.M.Polyakov: World Scientific;1990,Singapore.)

11. A.Alekseev and S.Shatashvili, Nucl.Phys. B323 (1989) 719.

12. S.Aoyoma, Int.J.Mod.Phys. A7 (1992) 5761.

13. V.G.Knizhnik and A.B.Zamolodchikov, Nucl.Phys. B247 (1984) 83 .

14. M.E.Peskin, SLAC Pub.4251, 1987. 